\title{
SOFIA: An innovative setup to measure complete isotopic yield of fission fragments
}

\author{
E. Pellereau ${ }^{1, a}$, G. Bélier ${ }^{1}$, G. Boutoux ${ }^{1}$, A. Chatillon ${ }^{1}$, A. Ebran ${ }^{1}$, T. Gorbinet ${ }^{1}$, B. Laurent ${ }^{1}$, \\ J.-F. Martin ${ }^{1}$, J. Taieb ${ }^{1}$, L. Audouin ${ }^{2}$, L. Tassan-Got ${ }^{2}$, B. Jurado ${ }^{3}$, H. Álvarez-Pol ${ }^{4}$, Y. Ayyad ${ }^{4}$, \\ J. Benlliure ${ }^{4}$, M. Caamaño ${ }^{4}$, D. Cortina-Gil ${ }^{4}$, B. Fernández-Domínguez ${ }^{4}$, C. Paradela ${ }^{4}$, \\ J.L. Rodríguez-Sánchez ${ }^{4}$, J. Vargas ${ }^{4}$, E. Casarejos ${ }^{5}$, A. Heinz ${ }^{6}$, A. Kelić-Heil ${ }^{7}$, N. Kurz ${ }^{7}$, \\ C. Nociforo ${ }^{7}$, S. Pietri ${ }^{7}$, A. Prochazka ${ }^{7}$, D. Rossi ${ }^{7}$, K.-H. Schmidt ${ }^{7}$, H. Simon ${ }^{7}$, B. Voss ${ }^{7}$, \\ H. Weick ${ }^{7}$, and J.S. Winfield ${ }^{7}$
}

${ }^{1}$ CEA, DAM, DIF, 91297 Arpajon, France

${ }^{2}$ CNRS, IPN Orsay, 91406 Orsay, France

${ }^{3}$ CNRS, CENBG, 33175 Gradignan, France

${ }^{4}$ Universidade de Santiago de Compostela, 15782 Santiago de Compostela, Spain

${ }^{5}$ Universidade de Vigo, 36310 Vigo, Spain

${ }^{6}$ Chalmers University of Technology, 41296 Gothenburg, Sweden

${ }^{7}$ GSI, Gesellschaft fur Schwerionenforschung GmbH, Planckstr. 1, 64291 Darmstadt, Germany

\begin{abstract}
We performed an experiment dedicated to the accurate isotopic yield measurement of fission fragments over the whole range. SOFIA exploits the inverse kinematics technique: using heavy ion beams at relativistic energies, fission is induced by Coulomb excitation in a high- $Z$ target. The fragments are emitted forward and both of them are identified in charge and mass. The setup will be presented, as well as preliminary spectra.
\end{abstract}

\section{Introduction}

Since its discovery seventy five years ago, the fission process have been intensively studied from many points of view. Neutrons, gamma and light-charged particles emission have been measured concurrently with fission yields to try to understand this complex phenomenom in which many aspects of both nuclear structure and nuclear dynamics seem to play correlated roles. Yet, today many unknowns remain. Traditionally, the experimental studies were done by bombarding actinide targets with neutrons. Since the 1980s, recoil-spectrometers such as Lohengrin have made a breakthrough in fission yield measurements [1] but the results still suffer from limitations. In particular, these studies are not able to provide charge yields with a sufficient resolution for the heaviest fission products. The target lifetime is also a severe restriction to the nuclei of interest and the back-to-back emission of the two fragments makes their simultaneous detection impossible. In constrast, the use of inverse kinematics has shown

\footnotetext{
ae-mail: j.eric.pellereau@cea.fr
}

This is an Open Access article distributed under the terms of the Creative Commons Attribution License 2.0, which permits unrestricted use, distribution, and reproduction in any medium, provided the original work is properly cited. 
extremely efficient to overcome those limitations. At GSI (Darmstadt, Germany) this technique gives access to a large variety of nuclei produced in-flight by a fragmentation reaction of a primary beam $\left({ }^{238} \mathrm{U}\right)$ and selected with a high-resolution recoil-spectrometer, the FRagment Separator (FRS) [2], to form a secondary beam. The experiment conducted in 1996 by K.-H. Schmidt et al. [3] has proved the feasibility of such a method. Fission of seventy different nuclei selected by the FRS, was studied. An unprecedent charge resolution was obtained for both fission fragments in coincidence, thanks to the relativistic energies which ensure the full stripping of all the fragments and their emission in a narrow forward angle. Collectively, other experiments in inverse kinematics were conducted at GANIL [4]. The latter provides heavy ion beams at lower energies than GSI. Therefore only one fragment is identified and a very good charge resolution is reachable in the light region. In contrast, the masses are much easier to obtain as compared to the GSI's experiments. Another noticeable difference is that the GSI provides nuclei below ${ }^{238} \mathrm{~Np}$ (included), while at GANIL, minor actinides can be produced by transfer reactions of a primary ${ }^{238} \mathrm{U}$ beam. SOFIA (Studies On FIssion with ALADIN) is the new-generation experiments conducted at GSI: it provides the charge and the mass identification for both fission fragments with a very good resolution and over the full range. This paper will present the experimental setup and a precise description of the detectors purposely designed for this experiment. In the last part, we will provide preliminary charge and mass spectra to illustrate the performances of the SOFIA setup.

\section{Experimental setup}

The SOFIA experiment was conducted in August 2012 in the experimental area Cave C at the GSI facility. Fission of many nuclei obtained with the FRS and ranging from ${ }^{183} \mathrm{Hg}$ to ${ }^{238} \mathrm{~Np}$ was studied. The fission is induced by Coulomb excitation in a high- $\mathrm{Z}$ target sitting in Cave $\mathrm{C}$, resulting in a transferred excitation function peaked at $11 \mathrm{MeV}$ for the compound nucleus. This is similar to a $5 \mathrm{MeV}$ neutron impinging a target in direct kinematics. Outgoing the target, the two fission fragments are analysed in charge $(Z)$ through energy loss measurement in a double-sided ionization chamber (the Twin MUSIC), and in mass (A) using a dedicaded recoil spectrometer developed by our collaboration to follow the $B \rho-T o F-\Delta E$ method. For each fragment the magnetic deflection in the large acceptance dipole (ALADIN), presents in Cave C, permits to access the mass number as shown in Eq. (1):

$$
B \cdot \rho \propto \frac{\gamma \cdot v \cdot A}{Z}
$$

where $B$ is the magnetic field value and $\rho, \gamma$, and $v$ are respectively the bending radius, the Lorentz factor and the velocity of the fragment. The bending radii $\rho$ are deduced from two horizontal positions measured by two Multi-Wire-Proportionnal-Counters (MWPCs), respectively situated upstream and downstream the magnet, and from the angle obtained with the Twin-MUSIC. The velocities of the fragments are deduced from a time of flight measurement over a 7.5 meters flight path. The setup is schematized in Figure 1.

\section{Detectors overview}

\subsection{Active target (AT)}

The active target is a stack of ionization chambers made of aluminum anodes and high- $Z$ cathodes (lead or depleted uranium) as illustrated on the left side of Figure 2. Fissions induced by Coulomb exchange between the incoming nuclei and a cathode are clearly idenfied by comparing the energy loss in two consecutive sections. Fission events occurring in other layers (mainly in plastic scintillators upstream from this detector) are only hot fission and can be suppress using the same principle, as seen in Figure 2. 


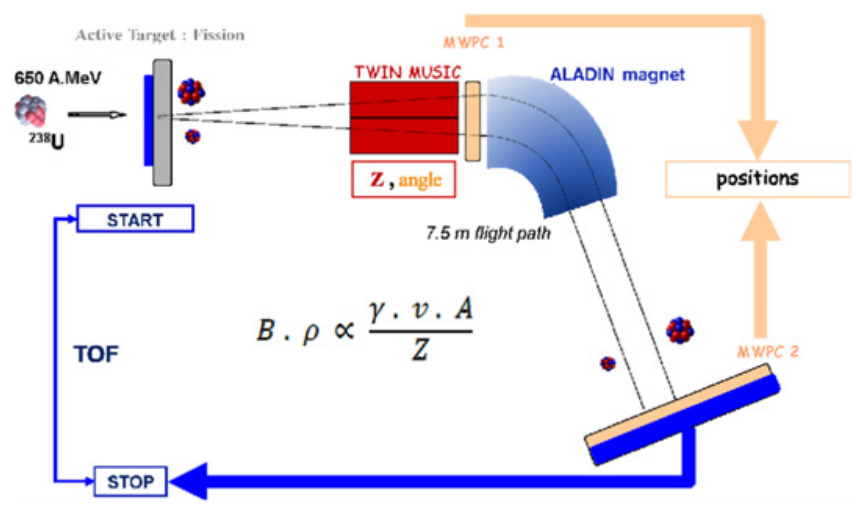

Figure 1. SOFIA setup.
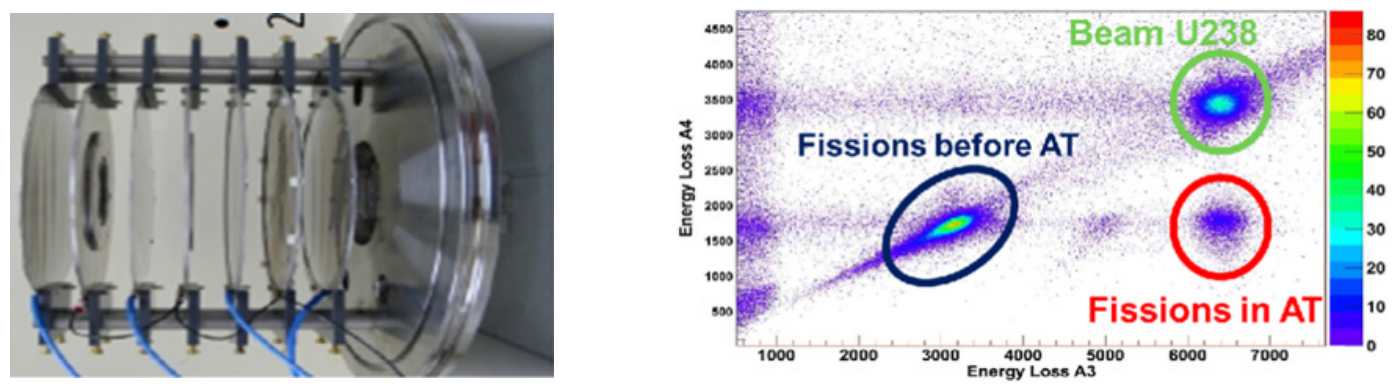

Figure 2. Left side: photography of the open active target. Right side: energy loss of two consecutive sections plotted with respect to each other. One can distinguish the primary beam (green circle), the fissions before the target (blue circle) and the fission events in the cathode between the two considered anodes (red circle).

\subsection{Twin-MUSIC: Charge and angle measurements}

The Twin-MUSIC is an ionization chamber of $60 \times 20 \times 20 \mathrm{~cm}^{3}$ active volume. As shown in Figure 3, a central cathode divides the volume into two parts, each one dedicated to a fission fragment. On both sides, the charge is deduced from the energy signals of ten anodes parallel to the common cathode. Thanks to the ten drift time measurements, they also provide the fragments angle in the horizontal plane right upstream the magnet. An excellent performance of this detector is of extreme importance. First, the charge resolution of the fission fragments is highly linked to the quality of the energy signals of this detector. Secondly, the angle reconstruction impacts the $B \rho$ value and therefore the mass resolution. To reduce the angular straggling, we chose a low- $\mathrm{Z}$ gas with Neon as a basis for the mixture instead of standard P10 (Argon 90\%, $\mathrm{CH}_{4}$ 10\%) gas. The drift velocity of the electrons in the gas (important for both pile-up and position resolution), their absorption and diffusion coefficients, as well as the sensibility to impurities were optimized by adding various components. Dedicated low noise charge preamplifiers have been especially designed to match the MUSIC signals characteristics. Each output is then connected to an electronic module MSCF-16 [6] which serves both as a spectroscopic amplifier (for the energy measurements) and a fast amplifier (to provide the timing signals, for the angle). The resolutions measured during the SOFIA experiment are of about $1.5 \%$ for Sn and $0.2 \mathrm{mrad}$, both FWHM, for the relative energy loss and the angle respectively. 


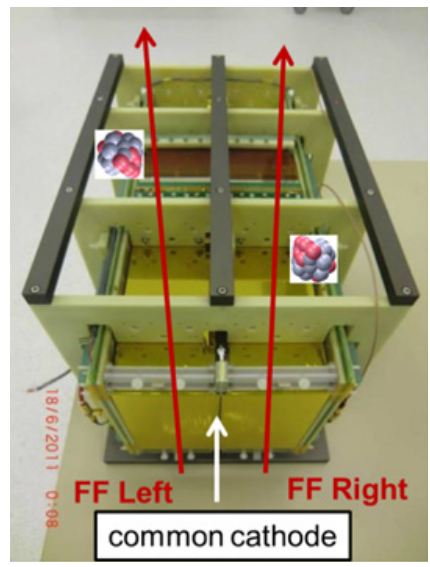

Figure 3. The Twin MUSIC (without its shielding box).
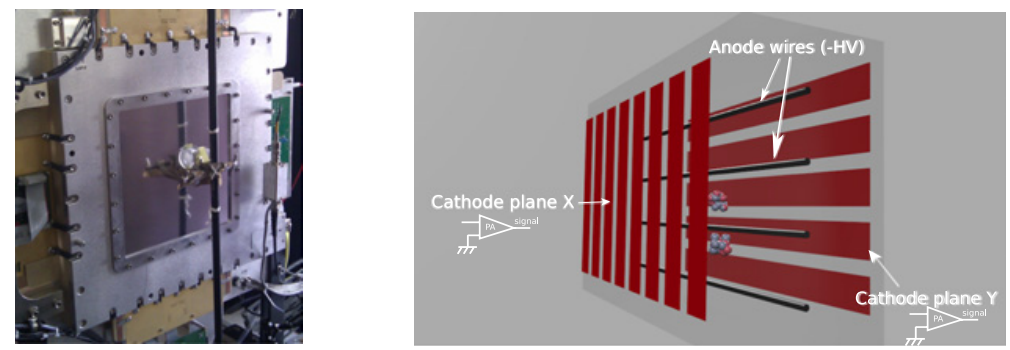

Figure 4. Left side: picture of the small MWPC. Right side: MWPC schematic representation. The X and Y planes sandwich the anodes wires and measure respectively the horizontal and vertical position.

\subsection{MWPCs: Position measurement}

The Multi-Wire-Proportionnal-Counters provide the horizontal positions of the two fission fragments needed for the bending radii measurements. Those two detectors are Cathode-Stripped Chambers (CSC) based on those designed for ALICE [5]. They provide excellent position resolution over wide dimensions using a reasonable number of electronic channels. They are $20 \times 20$ and $90 \times 60 \mathrm{~cm}^{2}$ in horizontal and vertical dimensions, upstream and downstream the magnet respectively. The position of the impact point is reconstructed from induced signals on cathode planes, resulting from the displacement of electrons avalanche close to the anodes wires.

For the SOFIA experiment, a cathode plane was also disposed parallel to the anode wires, as seen on the right side of the Figure 4, in order to measure the vertical positions. The gas employed in the two MWPCs was composed of Argon (80\%) and $\mathrm{CO}_{2}(20 \%)$ which acts as a quencher. The horizontal position resolutions are of 200 and $300 \mu \mathrm{m}$ FWHM for the first and second MWPC, respectively.

\subsection{Time of flight (ToF) wall}

All the technical choices relative to this detector are extensively described in [7] and only the main features are outlined here. The start detector only sees the primary beam (see Fig. 1) and is made of one small plastic scintillator ( $5 \mathrm{~cm}$ length). The Time-of-Flight wall size is $90 \times 60 \mathrm{~cm}^{2}$ to cover the full space occupied by the fission fragments at the end of the flight path. It is made of 28 plastic scintillators 

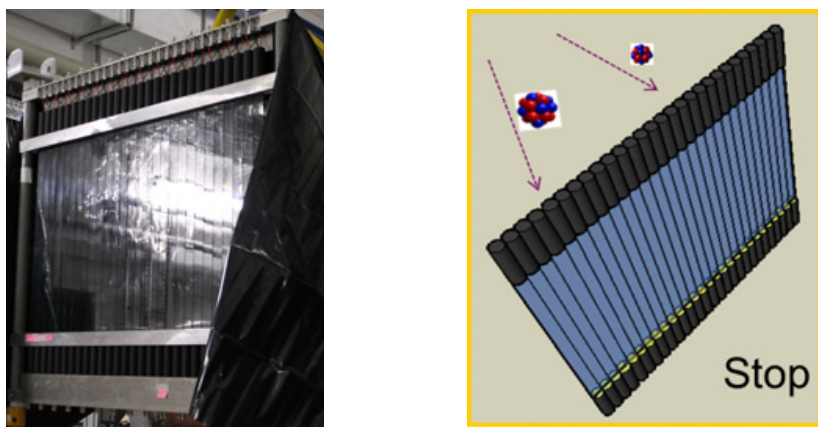

Figure 5. Left side: picture of the ToF wall detector. Right side: scheme of the detector. Photomultipliers are in grey and the scintillators in blue.
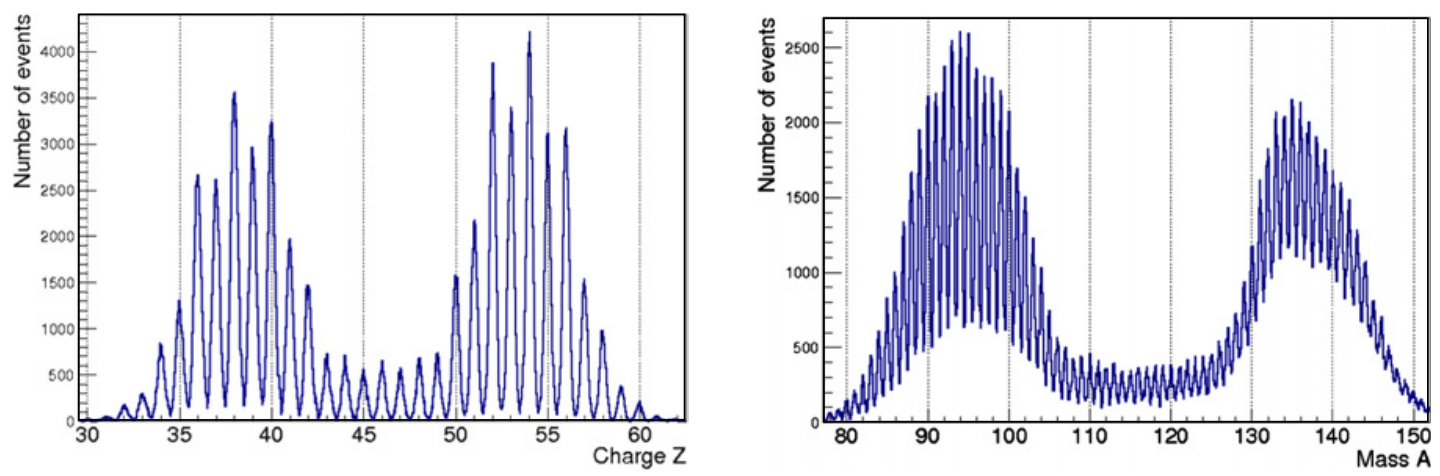

Figure 6. Preliminary charge (left side) and mass (right side) spectra for the electromagnetic fission of ${ }^{235} \mathrm{U}^{*}$.

( $3.2 \mathrm{~cm}$ wide, $60 \mathrm{~cm}$ long and $0.5 \mathrm{~cm}$ thick). This segmentation level is necessary at the end of the flight path to avoid the use of any light guides. All plastics are coupled to two photomultipliers tubes (PMTs) to provide a time measurement independent of the position. The stop detector is displayed in Figure 5. The needed ToF resolution to separate the neighbouring isotopes was estimated to be about $40 \mathrm{ps}$ FWHM. Such a good time resolution is required due to both the relativistic energies involved and the short flight path of $7.5 \mathrm{~m}$ only. The quality and the technical specificities of the plastic scintillators, photomultipliers, cables and electronic modules employed to build the ToF detection system permits to reach the required resolution. Dealing with relativistic heavy ions also plays a significant role in this achievement since the energy deposited in the plastics is always enormous. Because of the high number of electronic modules that would be needed, the time difference between the stop and the start detectors was not measured with TACs. Instead, we developed a new TDC module which profits from the Wave Union algorithm [8] implemented in field-programmable-gate-arrays (FPGA) and exhibits an intrinsic resolution of 17 ps FWHM.

\section{Preliminary results}

The charge and mass spectra of the fission fragments obtained for the ${ }^{235} \mathrm{U}^{*}$ are shown in Figure 6 . The corresponding resolutions are presented in Table 1. The isotopic separation is reached over the whole fragments range. 


\section{EPJ Web of Conferences}

Table 1. Resolutions FWHM in charge (left side) and mass (right side) in the light and the heavy regions.

\begin{tabular}{c|cc} 
Z & 38 & 54 \\
\hline Resolution (Z unit) & 0.44 & 0.37
\end{tabular}

\begin{tabular}{c|cc} 
A & 94 & 136 \\
\hline Resolution (A unit) & 0.58 & 0.82
\end{tabular}

\section{Conclusion}

The potential of inverse kinematics has been exploited in the SOFIA experiment to study the fission of a wide range of fissioning systems ranging from ${ }^{183} \mathrm{Hg}$ to ${ }^{238} \mathrm{~Np}$. Using a dedicated experimental setup with high resolution detectors, it has been possible, for the first time, to measure the complete distribution of the nucleons in the fission process.

\section{References}

[1] W.Lang, Nucl. Phys. A 345, 34-71 (1980)

[2] H. Geissel et al., Nucl. Instr. Meth. B 70, 286-297 (1992)

[3] K.-H. Schmidt et al., Nucl. Phys. A 665, 221 (2000)

[4] M. Caamaño et al., arXiv:1304.2647 (2013)

[5] C. Finck et al., Journal of Physics: Conference Series 50, 397 (2006)

[6] http://www.mesytec.com/

[7] A. Ebran et al., Nucl. Instr. Meth. http://dx.doi.org/10.1016/j.nima.2013.06.021

[8] http://www-ppd.fnal.gov/eedoffice-w/projects/ckm/comadc/PID765918.pdf 